\title{
COUPLED SIMULATION FOR FIRE-EXPOSED STRUCTURES USING CFD AND THERMO-MECHANICAL MODELS
}

\author{
Stanislav ŠUlc ${ }^{a, *}$, VÍt Šmilauer $^{a}$, FrantišEK WALD $^{b}$ \\ ${ }^{a}$ Czech Technical University in Prague, Faculty of Civil Engineering, Department of Mechanics, Thákurova 7, \\ 16629 Prague 6, Czech Republic \\ ${ }^{b}$ Czech Technical University in Prague, Faculty of Civil Engineering, Department of Steel and Timber Structures, \\ Thákurova 7, 16629 Prague 6, Czech Republic \\ * corresponding author: stanislav.sulc@fsv.cvut.cz
}

\begin{abstract}
Fire resistance of buildings is based on fire tests in furnaces with gas burners. However, the tests are very expensive and time consuming. This article presents a coupled simulation of an element loaded by a force and a fire loading. The simulation solves a weakly-coupled problem, consisting of fluid dynamics, heat transfer and mechanical model. The temperature field from the computational fluid dynamics simulation (CFD) creates Cauchy and radiative boundary conditions for the thermal model. Then, the temperature field from element is passed to the mechanical model, which induces thermal strain and modifies material parameters. The fluid dynamics is computed with Fire Dynamics Simulator and the thermo-mechanical task is solved in OOFEM. Both softwares are interconnected with MuPIF python library, which allows smooth data transfer across the different meshes, orchestrating simulations in particular codes, exporting results to the VTK formats and distributed computing.
\end{abstract}

KEYwORDS: Thermo-mechanical model, virtual furnace, computational fluid dynamics, multiphysics, fire resistance.

\section{INTRODUCTION}

Several single physical models exist for description of particular physical phenomena. Multi-physical coupled problems extend applications to more complicated tasks. In order to alleviate coupling among models, we created a python library which integrates solutions for computing simulations of structures exposed to fire. This paper presents approach how to interconnect single physical models.

\subsection{FiRE DYNAMICS ANALYSIS}

The issue of predicting fire process in a defined space domain is solved with the Fire Dynamics Simulator software (FDS) 11, developed at NIST. We modified the structure of the code to be able to call each computational time-step individually, we added several interface functions to get the dimensions of meshes, the temperatures in nodes, etc.

\subsection{Thermo-MEChANICAL ANALYSIS}

The thermo-mechanical analysis of the element takes place in OOFEM [2, 3] software. The thermal analysis uses a temperature field from FDS, which defines Cauchy and radiative boundary conditions on the element. Then, the solution of thermal analysis passes temperature to the mechanical analysis, which induces the thermal strain and modifies the material parameters as yield strength and elastic modulus. Temperature will also be used for timber structures where it controls carbonization process and changes the thermal conductivity.

\subsection{Adiabatic surface temperature}

The heat transfer to the construction is realized by convection and radiation. FDS can compute a quantity called "adiabatic surface temperature" (AST), which includes both convection and radiation influence. We compute the total heat flux $q_{\text {tot }}$ into the element with a method by Wickstrom [4] from the following equation:

$$
q_{\mathrm{tot}}=\varepsilon \sigma\left(T_{\mathrm{AST}}^{4}-T^{4}\right)+h\left(T_{\mathrm{AST}}-T\right)
$$

where $\varepsilon$ is the emissivity, $\sigma$ is the Stefan-Boltzmann constant and $h$ is the heat transfer coefficient. It allows us to realize the complicated task of radiation transfer very easily.

\section{InTERFACING FDS AND OOFEM USING MUPIF}

Interfacing FDS and OOFEM using MuPIF [5, 6] library, which provides several useful tools such as exporting data to VTU format, parallel computations and advanced handling with data fields and meshes. Both the FDS Fortran and OOFEM $\mathrm{C}++$ codes provide application interface for communication and they are compiled as shared libraries. Top-level steering script controls execution of both codes, data synchronization etc. The flowchart of the codes is given in Fig. 1

The FDS task consists of one or several uniform hexahedral meshes. In the beginning of the computation the geometries of all meshes are mapped from 


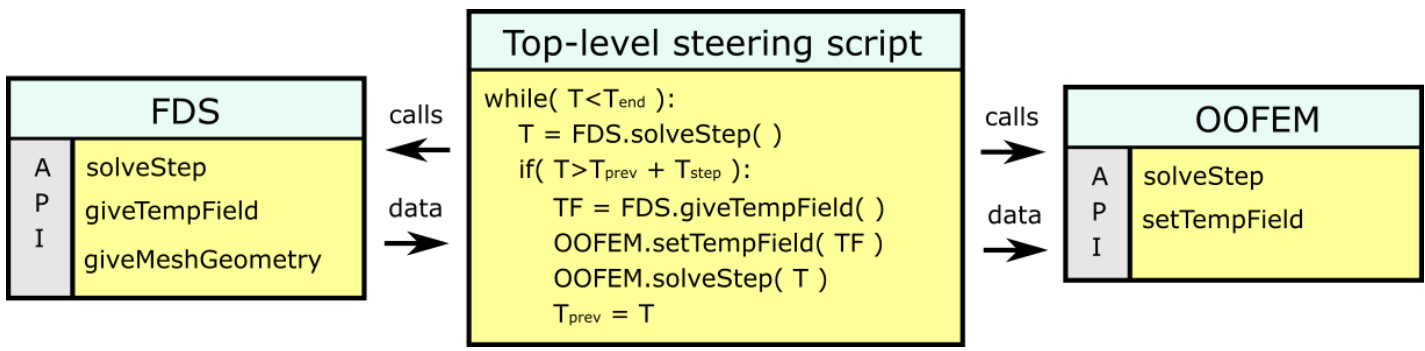

Figure 1. Flowchart of the codes.

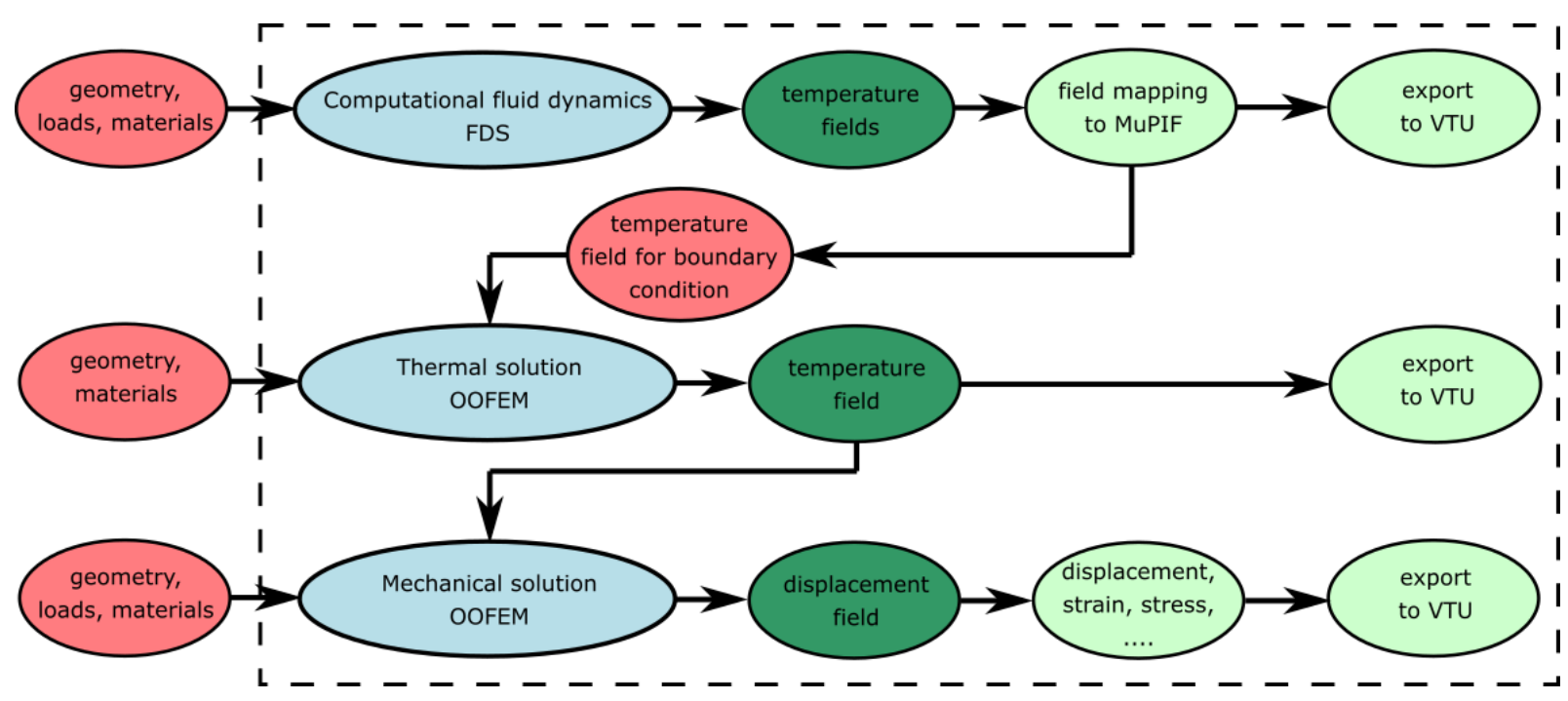

Figure 2. Data workflow.

FDS to MuPIF. The meshes contain discretized temperature field. We also load the coordinates of the points in which AST will be computed and create a mesh analogically. The only difference here is that the mesh consists only of points and has no elements. In this case, OOFEM uses no interpolation and uses a value of the closest point.

We note that the AST points should not be exactly on the element surface, they must be in the air close to the surface.

Here we describe the loop over one OOFEM timestep, see the data workflow in Fig. 2 and the flowchart in Fig. 1. First, FDS is called to process several computational steps until we reach the length of our defined time-step of the thermo-mechanical task. The FDS application determines its time-step itself and it is usually much shorter than the OOFEM time-step. Then, we load the temperature fields from FDS and copy them into the MuPIF fields. The field of AST values is copied into the OOFEM boundary-condition field. Then, OOFEM is asked to solve computational step of the thermal task and passes the computed temperatures of the construction to the mechanical task. The material parameters are updated due to the change of the temperature and the temperature strain is added. The mechanical response of the construction is obtained, which is the end of the computational loop.

As the FDS analysis is very time-consuming process and the computations can last couple of days, we use another MuPIF functionality to store the meshes with all the temperatures in all defined time-steps in files. In this regard, it is possible to precompute FDS simulation and compute multiple thermo-mechanical analyses separately and much faster.

Coarse FDS mesh can be refined in areas where structure elements occur. This improves precision in temperature predictions and gives more realistic results of the thermo-mechanical task.

The MuPIF library has a tool to export the loaded FDS meshes to VTU, so that the FDS temperature fields can be exported into this useful format. OOFEM exports its results to VTU format as well.

\section{EXAMPLE}

We demonstrate functionality of coupled CFD-thermomechanical model on a prototypical task, consisting of a steel beam placed into a furnace, see the geometry in Fig. 3. The furnace consists of two meshes. The bigger one covers the whole furnace and has 1000 cells, while the second one around the beam has 4800 cells. The âŮ́mesh structure is displayed in the results in Fig. 10. The inner space of the furnace is a cube with edge length $0.8 \mathrm{~m}$. The furnace is heated by 1 natural gas burners, placed in the middle of its floor. The output of the burner is $400 \mathrm{~kW}$ during the whole simulation. In the middle of the ceiling is a chimney. Both the holes are square-shaped with edge 


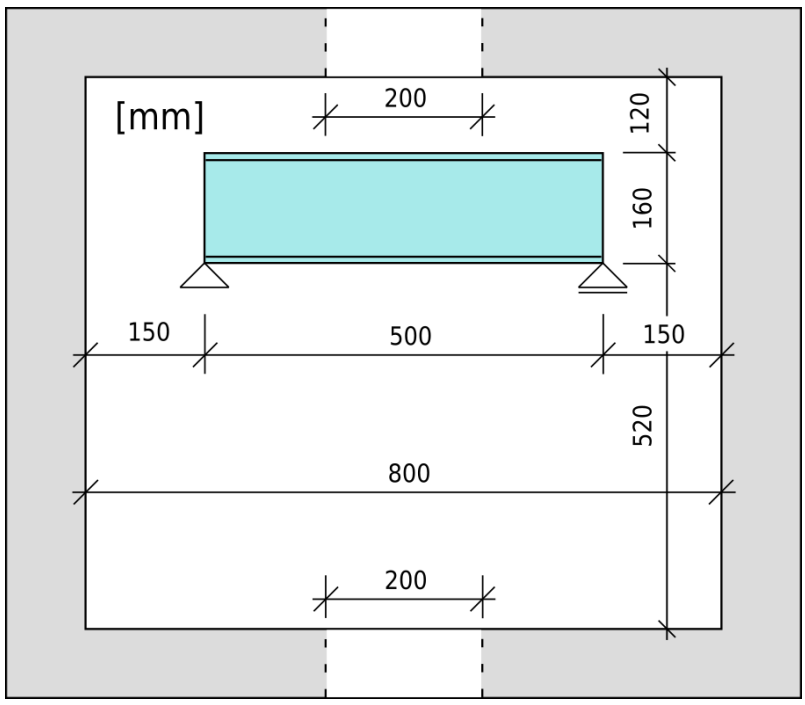

FiguRE 3. Furnace geometry.

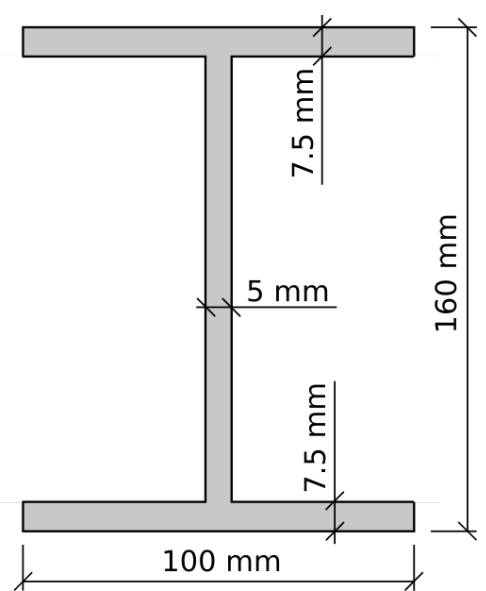

Figure 4. Cross-section of the beam.

size $0.2 \mathrm{~m}$. The simply-supported beam is made from steel I profile, see the geometry of its cross-section in Fig. 4. It is placed $0.52 \mathrm{~m}$ from the floor, horizontally in the centre. It is loaded only by its weight and the temperature. This example aims to the thermal task, because the used mechanical material model has constant parameters, non-dependent on temperature.

The material parameters are $E=200 \mathrm{GPa}, \nu=0.3$, $\rho=7850 \mathrm{~kg} / \mathrm{m}^{3}$ and $\alpha=12 \times 10^{-6}$. The length of the beam is $0.5 \mathrm{~m}$. Steel conductivity is displayed in Fig. 6 and capacity is displayed in in Fig. 7. Emissivity of the steel surface is 0.8 and the heat-transfer coefficient is $20 \mathrm{~W} \cdot \mathrm{m}^{-2} \cdot \mathrm{K}^{-1}$.

The OOFEM model of the beam consists of 540 cells, which is enough for our purpose, see Fig 5

The results were computed end exported to VTU files. Fig. 10 shows a cross-section through the middle of the furnace. We can see the two FDS meshes, one coarse on the whole furnace and one finer around the beam. Detailed temperatures of the beam are displayed in Fig. 11, the stress magnitude $\sigma_{\max }$ is in Fig. 13.

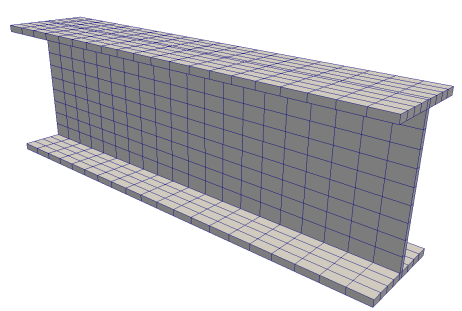

Figure 5. OOFEM mesh of both thermal and mechanical task.

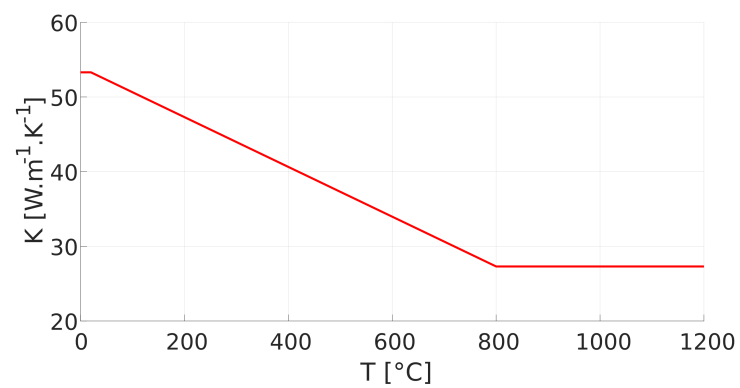

Figure 6. Steel conductivity.

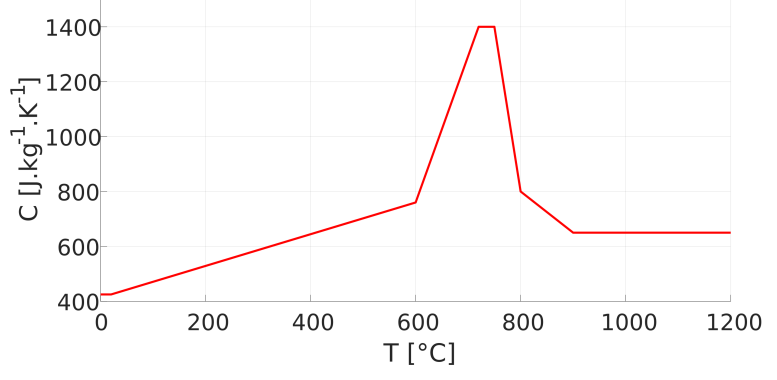

Figure 7. Steel capacity.

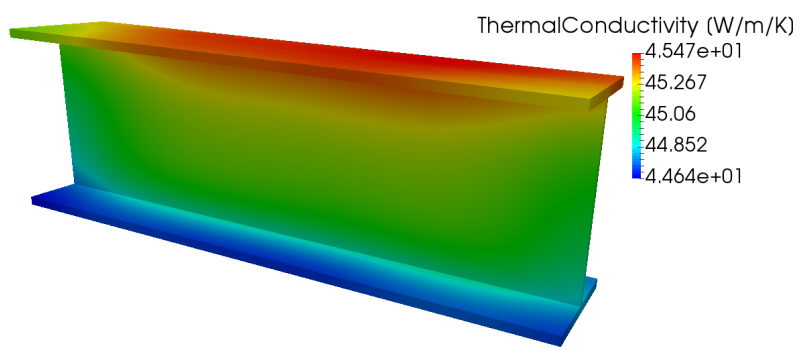

Figure 8 . Thermal conductivity at $\mathrm{t}=480 \mathrm{~s}$.

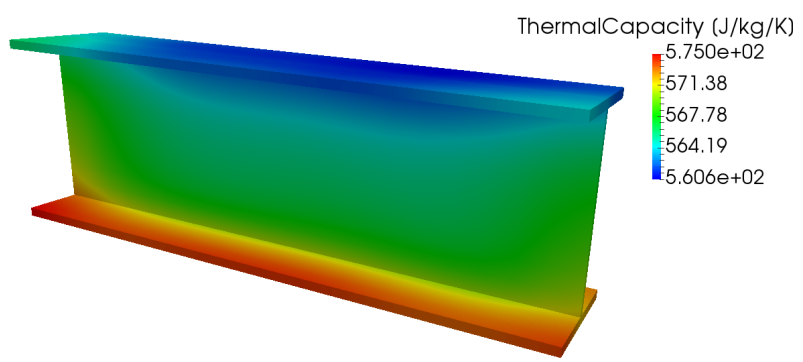

Figure 9. Thermal capacity at $\mathrm{t}=480 \mathrm{~s}$. 


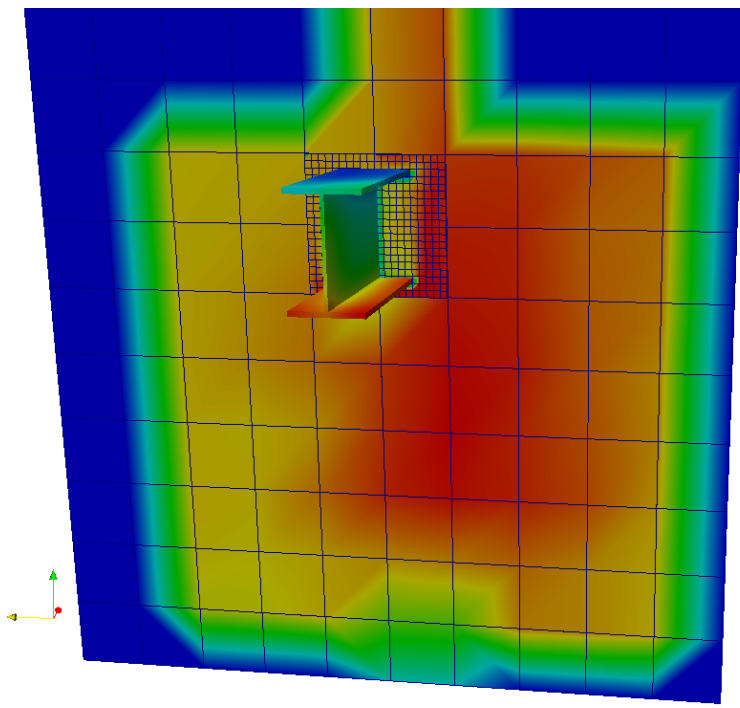

Element_Temperature $\left({ }^{\circ} \mathrm{C}\right)$

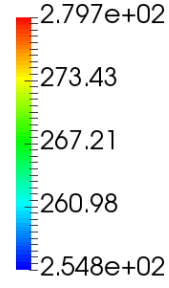

AlR_Temperature $\left({ }^{\circ} \mathrm{C}\right)$

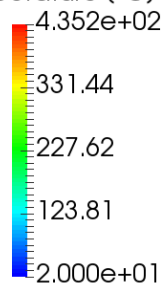

Figure 10. Temperatures at $\mathrm{t}=480 \mathrm{~s}$, section in the middle of the furnace.

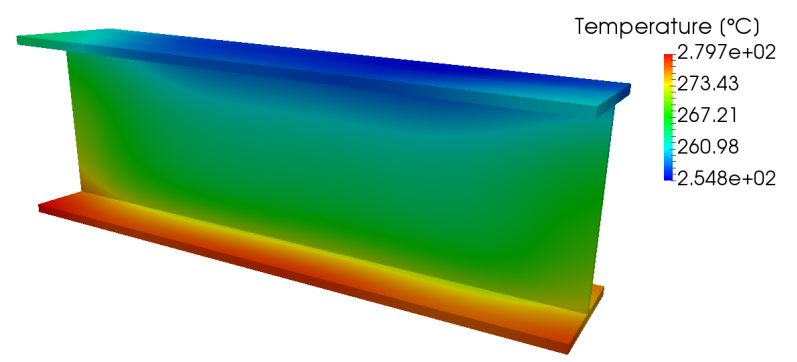

Figure 11. Temperature at $\mathrm{t}=480 \mathrm{~s}$.

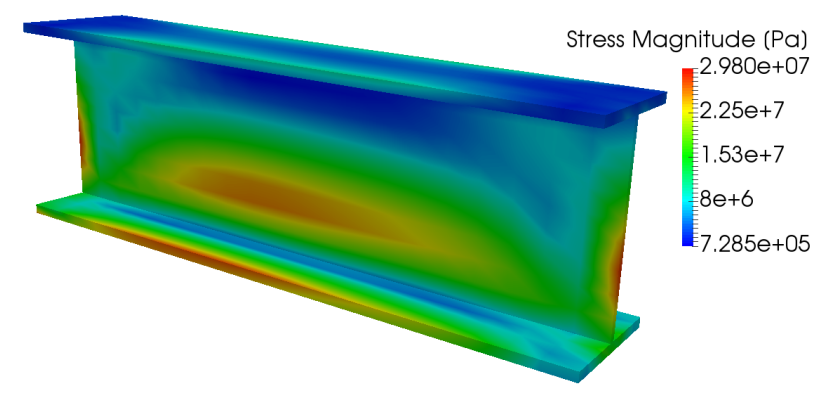

FiguRE 12 . Stress at $\mathrm{t}=480 \mathrm{~s}$.

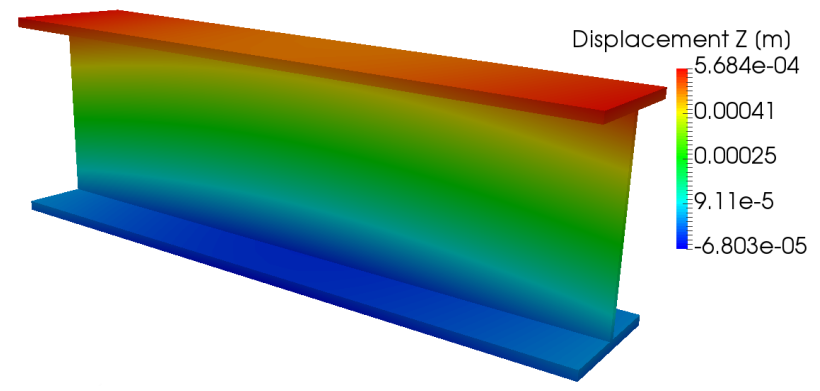

Figure 13. Displacement $\mathrm{z}$ at $\mathrm{t}=480 \mathrm{~s}$.

\section{Conclusions}

This paper presents an approach for computing weakly-coupled problems, focusing on computational fluid dynamics coupled with thermo-mechanical analysis. Temperature field from FDS was validated previously [7, performance of structure elements presents a consecutive step. Validation of this multi-physical approach happens during 2017 on steel and timber and concrete structural elements.

\section{ACKNOWLEDGEMENTS}

We gratefully acknowledge the financial support from the Czech Technical University in Prague, the grant SGS17/042/OHK1/1T/11.

We also gratefully acknowledge the financial support from the Czech Science Foundation, the grant GACR $16-18448 \mathrm{~S}$

\section{REFERENCES}

[1] NIST. FDS, Fire Dynamics Simulator, project home page, 2017. http://www.pages.nist.gov/fds-smv/

[2] B. Patzák. OOFEM project home page, 2000. http://www. oofem.org/

[3] B. Patzák. OOFEM - an object-oriented simulation tool for advanced modeling of materials and structures. Acta Polytechnica 52(6):59-66, 2012.

[4] U. Wickstrom, D. Duthinh, K. McGrattan. Adiabatic Surface Temperature for Calculating Heat Transfer to Fire Exposed Structures. In Proceedings of the Eleventh International Interflam Conferrence, Interscience Communications. London, 2007.

[5] B. Patzák. MuPIF project home page, 2010. http://www.mech.fsv.cvut.cz/mupif//

[6] B. Patzák, V. Smilauer, G. Pacquaunt. Design of a Multiscale Modelling Platform. In 15th International Conference on Civil, Structural, and Environmental Engineering Computing. Prague, Czech Republic, 2015.

[7] K. Cábová, V. Asgf, G. Sdfggf. Modelling of Standard Fire Test. In Engineering Mechanics. Svratka, Czech Republic, 2017. 